\title{
Research Paper \\ Effect of Intracerebroventricular Morphine Withdrawal on Anxiety Behavior in Male Rats Reared in Social Isolation
}

\author{
Ghasemali Khodabandeh ${ }^{1} \odot{ }^{*}$ Gholamhassan Vaezi $^{1} \odot$, Vida Hojati ${ }^{1}$, Sharam Sharafi ${ }^{1}$
}

1. Department of Biology, Faculty of Basic Science, Damghan Branch, Islamic Azad University, Damghan, Iran.

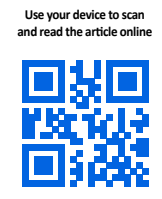

Ctration Khodabandeh Gh, Vaezi Gh, Hojati V, Sharafi Sh. [Effect of Intracerebroventricular Morphine Withdrawal on The Anxiety Behavior in Male Rats Reared in Social Isolation (Persian)]. Quarterly of "The Horizon of Medical Sciences". 2019; 25(4):352363. https://doi.org/10.32598/hms.25.4.352

https://doi.org/10.32598/hms.25.4.352

Key words:

Anxiety, Morphine, Social isolation, Rats

\section{ABSTRACT}

Received: 25 Feb 2019

Accepted: 23 Aug 2019

Available Online: 01 Oct 2019
Aims Narcotics prescription has controversial effects on the occurrence of anxiety processes; however, its acute and chronic effects on behavioral differences in social isolation are unclear in the processes of dependence and withdrawal. The present study aimed to investigate the effects of acute and chronic intracerebroventricular morphine sulfate withdrawal on the fear and anxiety behaviors of male rats reared in social isolation.

Methods \& Materials The present experimental study investigated 32 male 21-day-old male weaned Wistar rats that were divided into two groups of saline (control) and morphine receivers (test). They were then divided into acute and chronic subgroups that were reared under social isolation conditions. The rats of the acute daily consumption group received $10 \mu \mathrm{g} / \mathrm{kg}$ of morphine sulfate solution via intracerebroventricular injection for 10 days, but the chronic rats received it for 60 days. After the end of dependence by its withdrawal, the rats were quitted for 5 days, and their anxiety levels were measured using the Elevated Plus Maze (EPM). The obtained data were analyzed in SPSS using One-Way Analysis of Variance (ANOVA), Tukey's posthoc test and Paired Samples t-test.

Findings The research results indicated that the percentage of time and number of open arm entries in rats reared in social isolation significantly decreased during the dependence phase and 5 days after withdrawal in acute and chronic groups $(P<0.001)$. Furthermore, their anxiety rate increased compared to the control group. The findings also suggested a higher incidence of anxiety among chronic consumer groups than acute consumer groups after abstinence.

Conclusion The study findings indicated that the discontinuation of morphine consumption in social isolation could increase the incidence of anxiety behaviors in rats. Therefore, negative emotional states associated with acute and chronic morphine withdrawal could lead to anxiety-like behaviors.Keywords: Anxiety, Morphine, Social isolation, Rats.

\section{* Corresponding Author:}

Gholamhassan Vaezi, PhD.

Address: Department of Biology, Faculty of Science, Damghan Branch, Islamic Azad University, Damghan, Iran.

Tel: +98 (912) 9178644

E-mail: gh.vaezi@yahoo.com 


\section{Extended Abstract}

\section{Introduction}

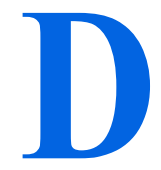

rug addiction is a type of chronic and regressive brain disease characterized by loss of control over medication use, repeated attempts in quitting or reducing drug use, drug use continuation despite negative consequences, reduced engagement in social, occupational, and social activities. They, instead, continue seeking for drug, self-prescription, and the emergence of departure or withdrawal symptoms $[1,2]$. Symptoms such as muscle aches, severe muscle irritability, hostility, convulsions, diarrhea, and anxiety become so painful and intolerable that in many cases the patients resume drug use $[4,5]$.

Part of the soothing effect of opioids is through the predictable reduction of anxiety [6]. Previous studies have shown that morphine dependence in addicted mice causes anxiety-like behaviors [7]. Anxiety is one of the most commonly reported symptoms of quitting a variety of drugs. It is thought that termination of emotional withdrawal symptoms such as anxiety, numbness, agitation, restlessness, and fatigue are to be the drivers of resuming drug use that eventually lead to drug dependence [8, 9]. Discontinuation of opioids is associated with anxiety-like symptoms in humans and animals [9]. Animal models show similarities between drug dependence and sedation at behavioral and neurochemical levels [8].

\section{Methods}

For this experimental study 32 male Wistar rats that weaned aged 21 days were used. Then each rat was kept in a separate cage measuring $22 \times 25 \times 40 \mathrm{~cm}$, in a state of social isolation. Specific water and food (pellets) were readily available, except during testing. The animals were housed in a $12 \mathrm{~h}$ light-dark period (seven in the morning to seven in the night) and $22 \pm 2^{\circ} \mathrm{C}$ without any sound or noise pollution. The experiments were carried out from 9 am to $1 \mathrm{pm}$.

Rats were randomly divided into four groups, each with eight rats. Rats were divided into control (saline) and Morphine Sulfate (Sigma-Germany) groups, each containing acute and chronic subgroups. In acute groups, the intravenous-cerebral injection was performed for 10 days and in chronic groups for 60 days [7, 19]. Five days after discontinuation of drug use, in the acute groups on days 15 and in the chronic groups on days 65 , the maze tests were performed.

Morphine sulfate was injected intracerebroventricular at a dose of $10 \mu \mathrm{g} / \mathrm{kg}$. All injections were performed in 100 rg volume and $0.1 \mathrm{ml}$ on body weight.

\section{Results}

The results of this study showed that five days after discontinuation of intracerebroventricular administration of morphine, the attendance percentage in the open arm of

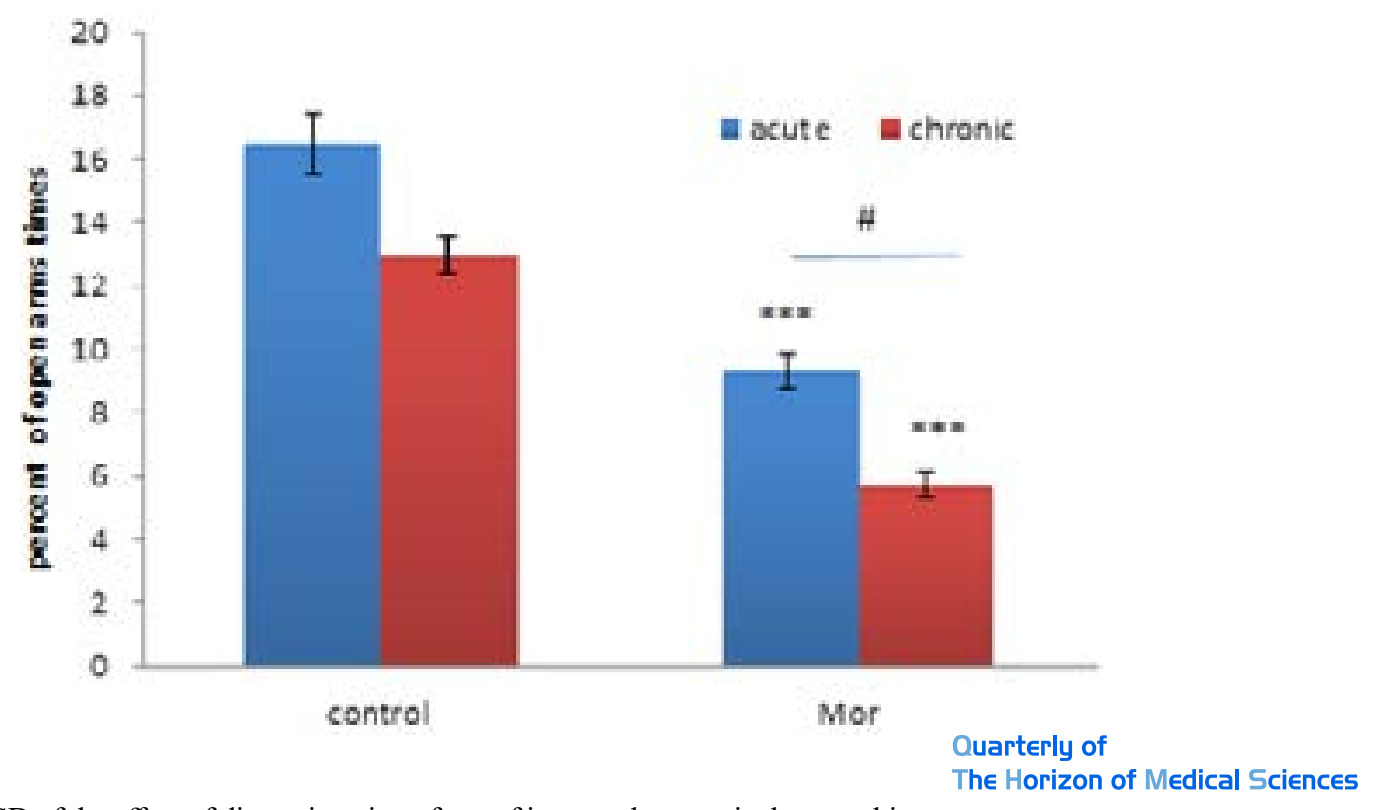

Figure 1. Mean $\pm \mathrm{SD}$ of the effect of discontinuation of use of intracerebroventricular morphine

$(10 \mu \mathrm{g} / \mathrm{kg}$, Mor $)$ in precentage of presence in open arm in plus maze in rats under social isolation compared to control. $\mathrm{P}<0.05$ : significant difference with control group receiving saline. : $\# \mathrm{P}<0.05$ significant difference between acute and chronic groups 


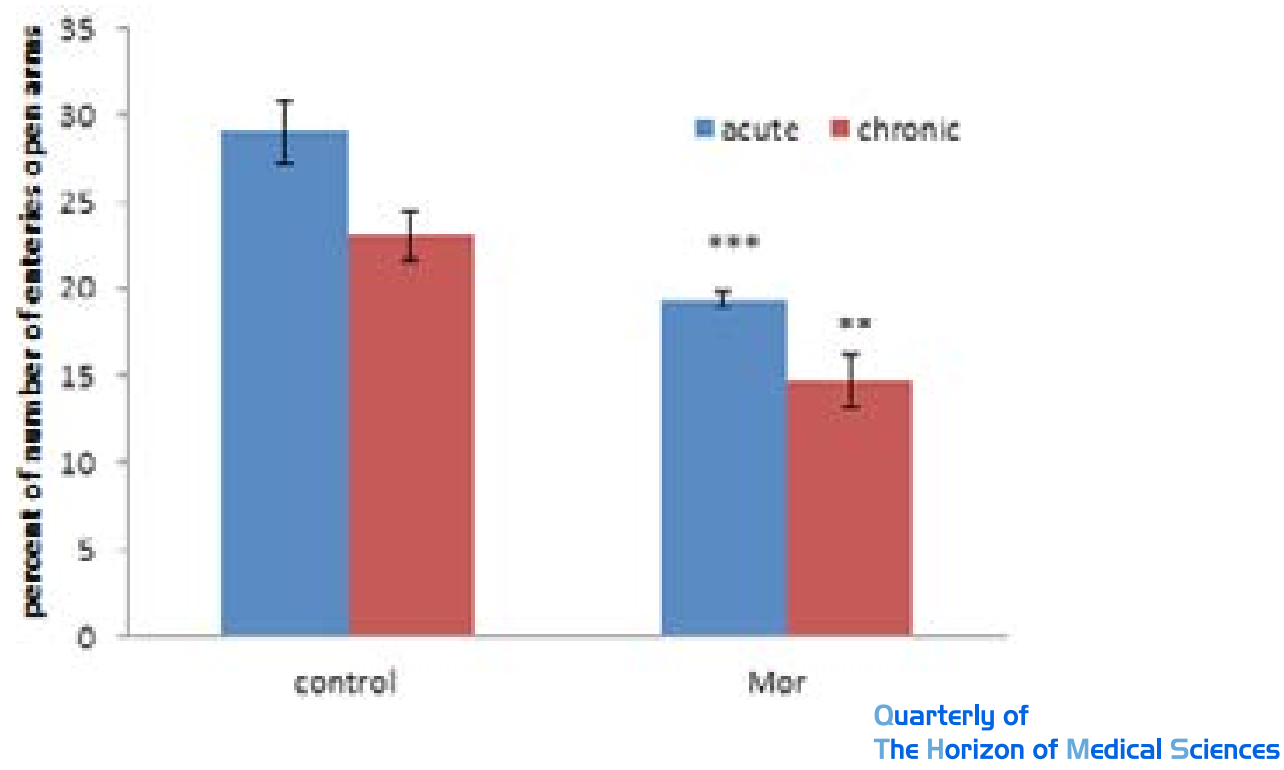

Figure 2. Mean $\pm \mathrm{SD}$ of the effect of discontinuation of use of intracerebroventricular morphine

$(10 \mu \mathrm{g} / \mathrm{kg}$, Mor) in precentage of presence in open arm in plus maze in rats under social isolation compared to control.

$* * * \mathrm{P}<0.01 * *, \mathrm{P}<0.001$ : Significant difference with control group receiving saline. $\mathrm{P}<0.05$. \#: Significant difference between acute and chronic groups

Equine Protozoal Myeloencephalitis (EPM) was significantly decreased in acute and chronic groups compared to the control group $(\mathrm{P}<0.001)$ (Figure 1). The percentage of the entrance to the open arm of EPM in the acute and chronic groups was significantly reduced compared to the control group $(\mathrm{P}<0.001)$ (Figure 2$)$. However, there was no significant difference in the entrance percentage in the closed arm of EPM compared to the control group (Figure 3).

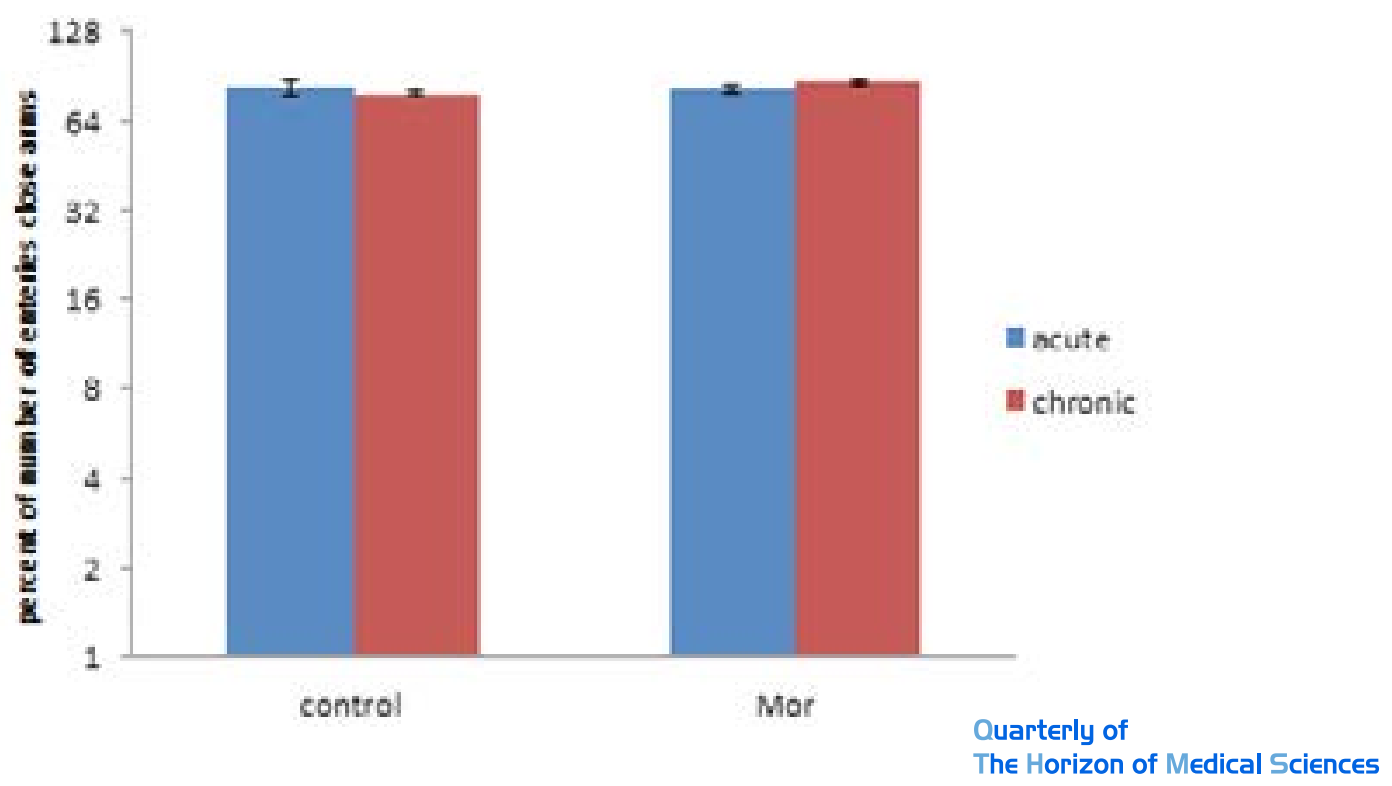

The comparison between acute and chronic groups showed a significant decrease in the attendance percentage in EPM in the chronic groups compared to the corresponding acute group $(\mathrm{P}<0.05)$ (Figure 1), but the percentage of open and closed arm entrance and the association between acute and chronic groups was not significant (Figures 2 and 3).

\section{Discussion}

The Horizon of Medical Sciences

Figure 3. Mean \pm SD of the effect of discontinuation of use of intracerebroventricular morphine

$(10 \mu \mathrm{g} / \mathrm{kg}$, Mor) in precentage of presence in closed arm in plus maze in rats under social isolation compared to control. $\mathrm{P}<0.05$ : Significant difference with control group receiving saline. : $\# \mathrm{P}<0.05$ significant difference between acute and chronic groups 
The results showed that intracerebroventricular morphine-treated groups in acute and chronic conditions in rats cultivated under social isolation, five days after quitting morphine sulfate, compared to control groups, the percentage, and the number of the entrance to open arm of EPM declined.

Compared between the acute and chronic groups, in the chronic groups, after quitting, the percentage reduction of the entrance to EPM was higher but not significant; thus, morphine dependence and also quitting it can affect the rate of the rats' anxiety.

The acute and chronic administration of morphine was significantly different between the groups receiving morphine and the control group, indicating a type of morphine dependence and addiction in mice. Our findings indicate that quitting or dependent morphine-treated rats are more anxious than the control group; this mode in chronically addicted rats is often higher than in acute-treated rats.

\section{Conclusion}

The results of this study showed that discontinuation of acute and chronic intracerebroventricular administration of morphine under social isolation can increase the mode of anxiety behavior in rats. The rate of anxiety behavior in rats in acute administration was lower than in chronic administration at the time of dependence and after quitting. In summary, negative affective states associated with acute and chronic morphine quitting comprised anxietyrelated behavior.

Animal models of negative affective consequences of opioid quitting should be useful in outlining the neurochemical and neuro-anatomical substrates of brain circuits related to stress, affective states, and brain reward. The use of social isolation after weaning among rats is useful for creating behavioral outcomes similar to the results of unpleasant experiences of isolation in humans and is the potential to provide preclinical findings that may turn into clinical research.

Since few studies have been performed on female mice, it is suggested to investigate the effect of acute and chronic morphine sulfate quitting on fear and anxiety behavior in female rats in social and non-social contexts, according to different injection methods.

\section{Ethical Considerations}

\section{Compliance with ethical guidelines}

This study has obtained its ethical approval from the Research Ethics Committee of Islamic Azad University of Damghan Branch (code: IR. REC. 1396.1544). All experiments on animals in this study was according to the Guide for the Care and Use of Laboratory Animals.

\section{Funding}

This study received no financial support from any organization.

\section{Authors' contributions}

Conceptualization, methodology, and investigation by Ghasemali khodabandeh; writing by Gholamhassan Vaezi; writing, methodology and data analysis by Vida Hojati; consulting by Sharam Sharafi.

\section{Conflicts of interest}

The authors declare no conflict of interest related to the present manuscript.

\section{Acknowledgements}

The authors would like to thank participants and Islamic Azad University of Damghan Branch for their valuable cooperation. 
This Page Intentionally Left Blank 


\title{
بررسى تأثير قطع مصرف درونبطنى ـ مغزى مرفين بر رفتار اضطراب در رتهاى نر يرورشيافته

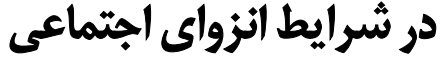

\author{
قاسمعلى خدابنده' ـ "غلامحسن واعظى' •، ويدا حجتى'، شهرام شرفى' \\ ا. كروه زيستشئاسى، دانشكده علوم بايه، واحد دامغان، دانشكاه آزاد اسلامى، دامغان، ايران.
}

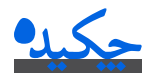

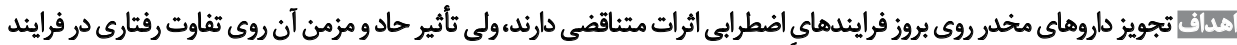

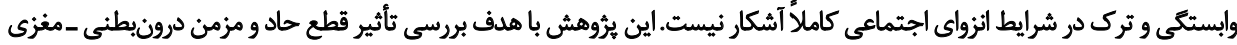

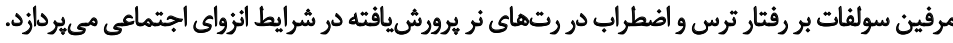

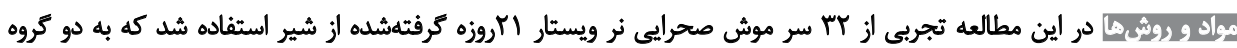

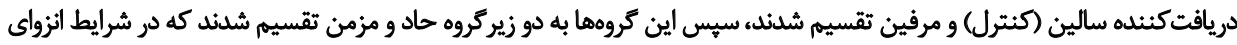

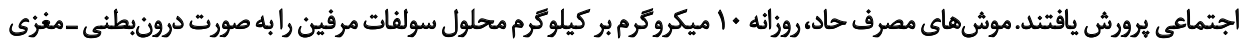

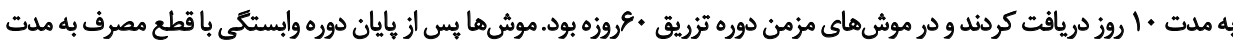

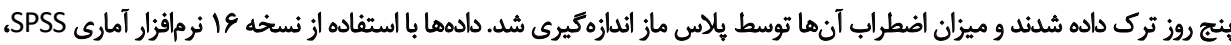

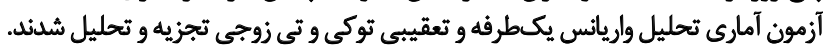

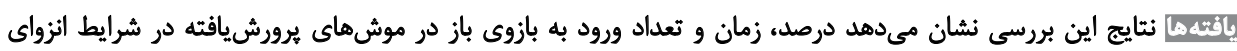

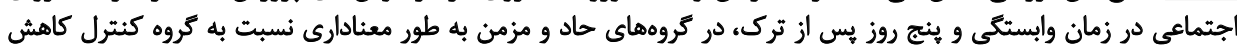

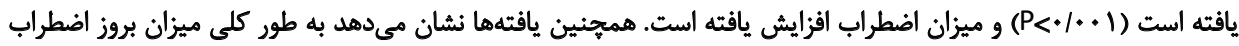

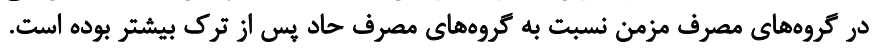

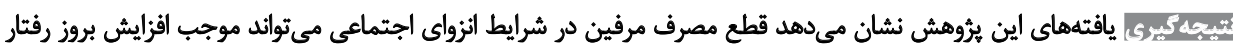

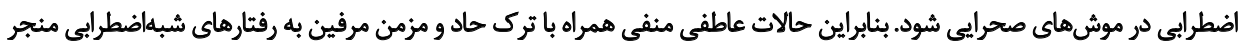

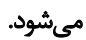

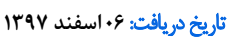

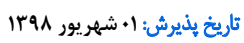

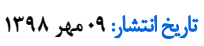

كليدوارهها:

اضطراب، مرفين، انزواى

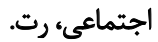

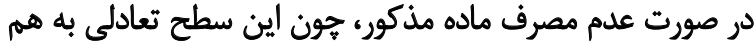

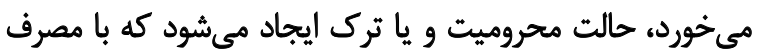

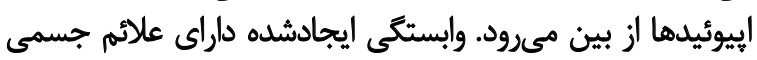

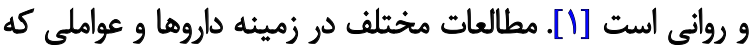

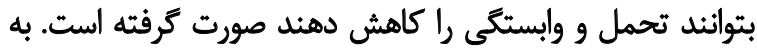

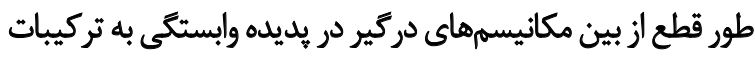

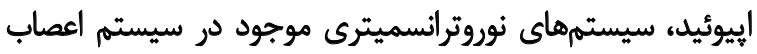

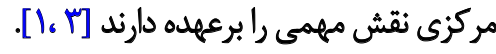

هنكام قطع مصرف مواد مخدر و در حين تركى، علائمى تحري

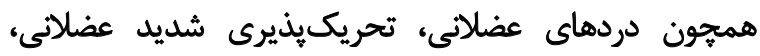

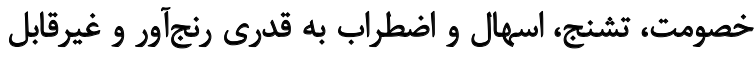

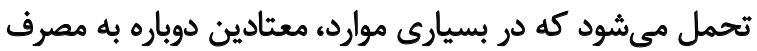

مقدمه

اعتياد به مواد مخدر يك نوع بيمارى مزمن عودكننده مغز

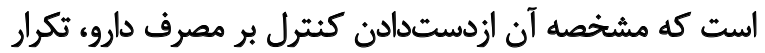

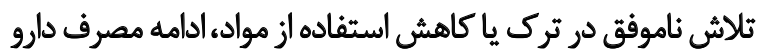

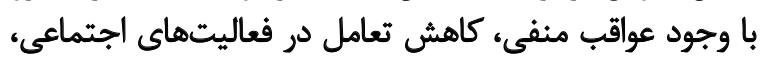

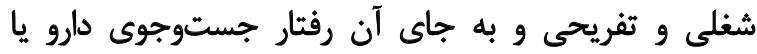

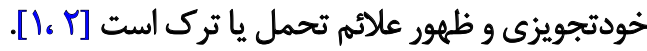

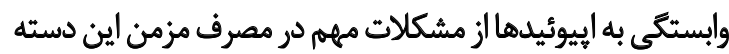

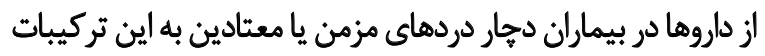

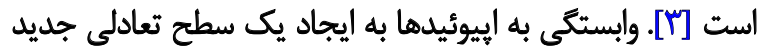

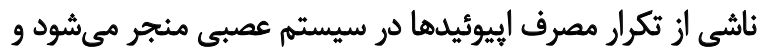

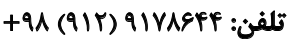
يست الكترونيكي: gh.vaezi@yahoo.com 
درباره آن انجام شده است و شرايط محيطى در تغيير حساسيت

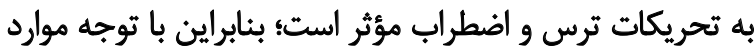

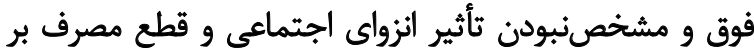

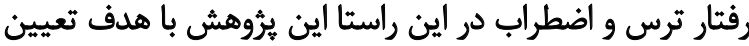

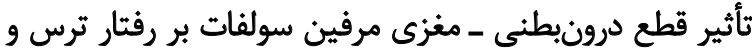

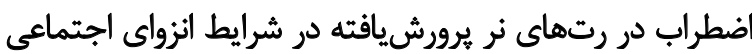
انجام شده است.

مواد وروش

براى انجام اين تحقيق تجربى از זب سر موش صحرايى نر نثراد

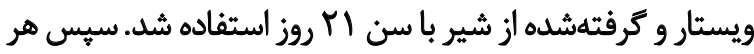

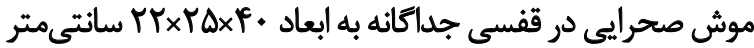

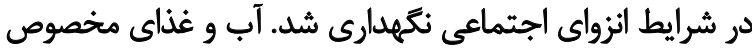

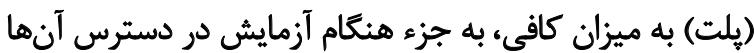

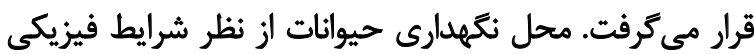

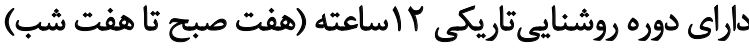

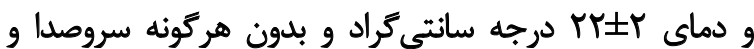

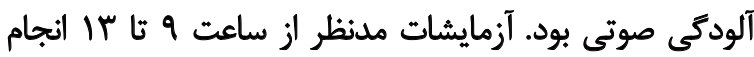

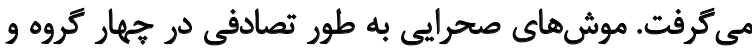

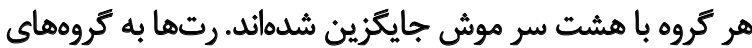

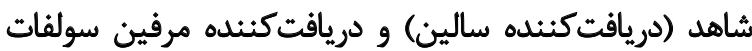

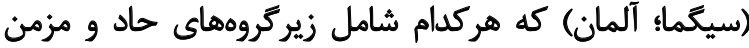

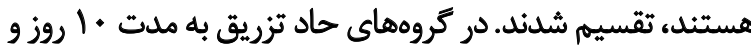

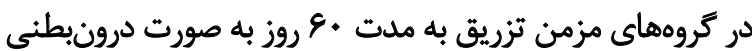

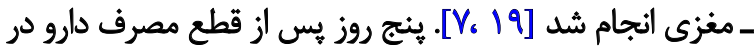

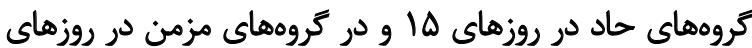

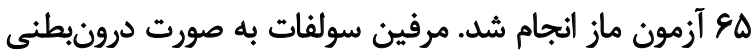

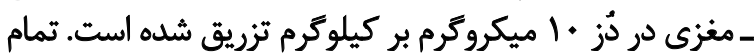

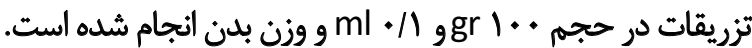

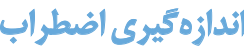

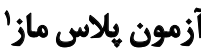

براى اندازميرى رفتارهاى اضطرابى و بررسى فعاليت حركتى

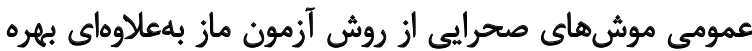
يركه شم.

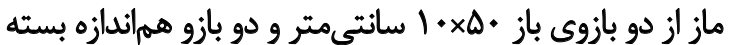

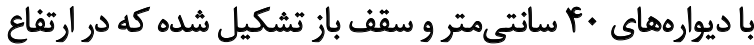

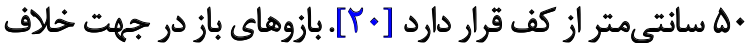

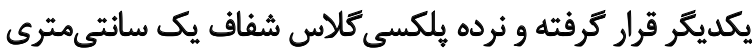

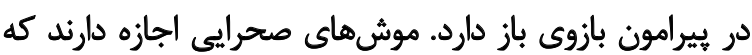

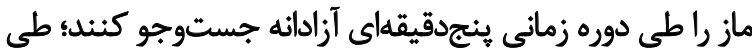

1. Elevated Plus-Maze Test (EPM)

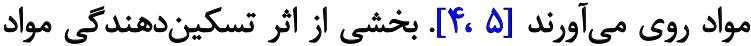

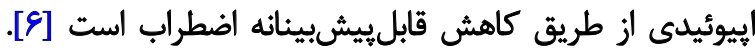

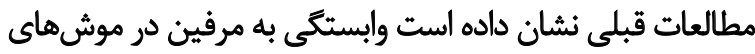

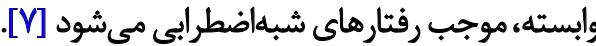
اضطراب يكى از معمولترين علاثم كزارششده از قطع مصرف

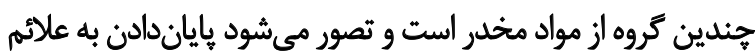

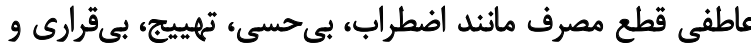

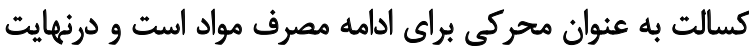

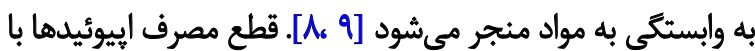

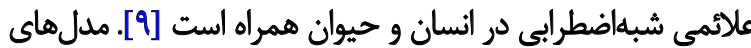

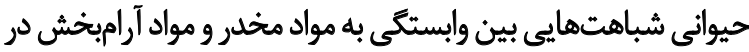

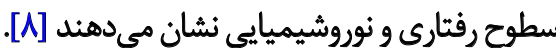
عوامل محيطى يكى از عواملى هستند كه باسخ به محركها

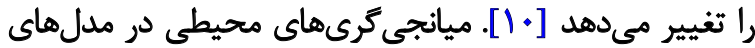

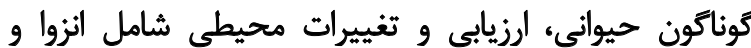

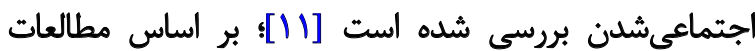

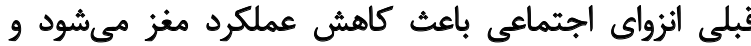

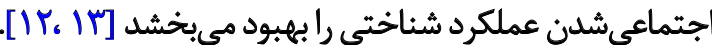
در مطالعات، تغييرات عملكرد مغز همراه با نروزثز بررسى

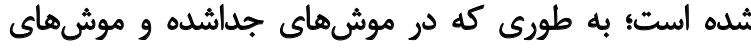

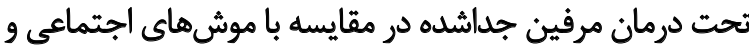

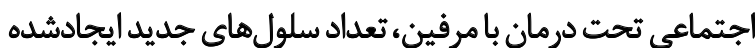

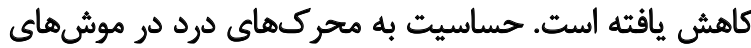

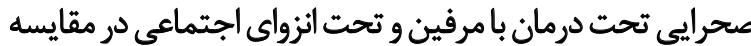

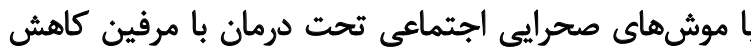

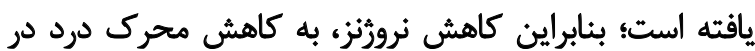

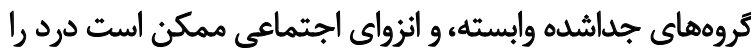

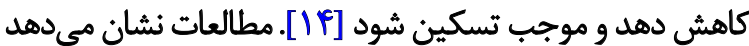

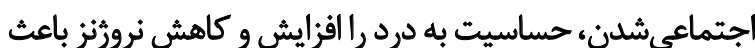

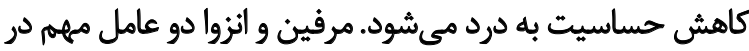
كاهش حساسيت به درد و كاهش نرورثز هستند [10]. تأثير انزواي زودهنكام بر كيرندههاي إيوئيدى بررسى شده

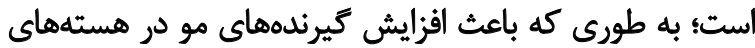

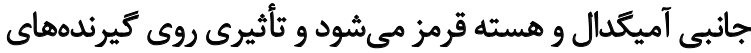

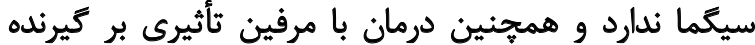

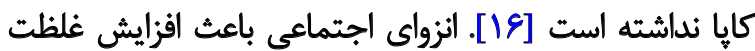

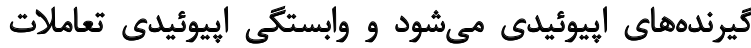

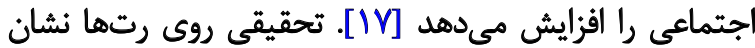

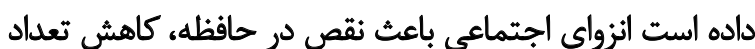

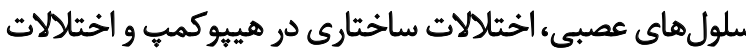

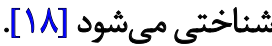

رفع ترس و اضطراب يكى از مسائلى است كه تلاشهاى زيادى 
با مقايسه بين كروههاي حاد و مزمن مشخص شد در درصد

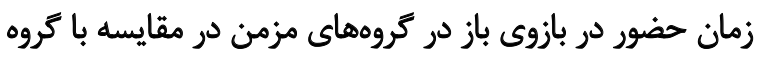

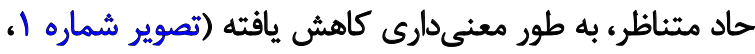

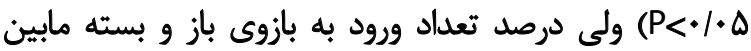

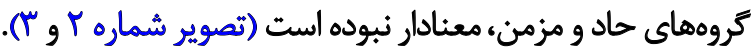

ث

نتايج اين تحقيق نشان داده است در زروههاى دريافتكنيده

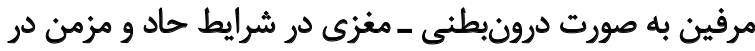

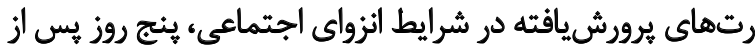

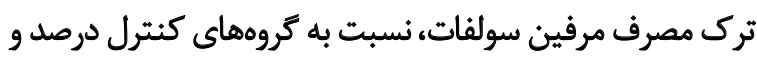
تعداد ورود به بازوى باز EPM كاهش يأقته است. در مقايسه بين بين

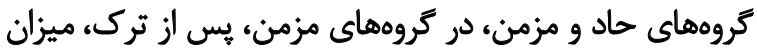

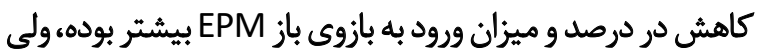

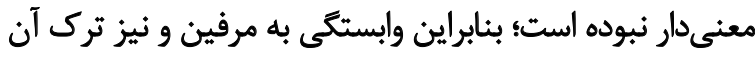

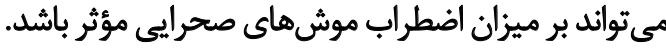
تجويز حاد و مزمن مرفين بين كروههاي دريافت كننده مرفين

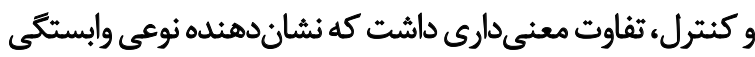

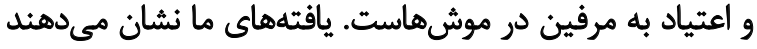

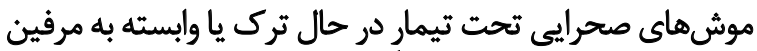

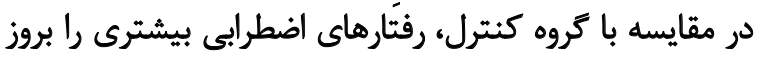

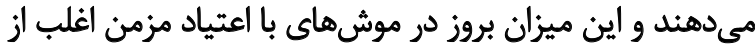

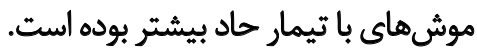

در اين مطالعه نشان داده شد يس از ترك نيز حيوانات

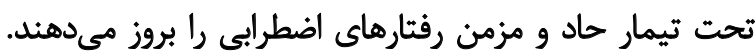

اين زمان، تعداد كل ورودها به بازوهاي باز و بسته و نيز زمان

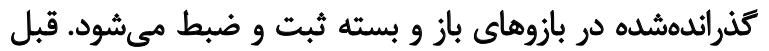

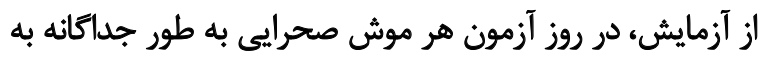

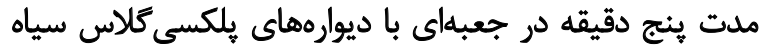

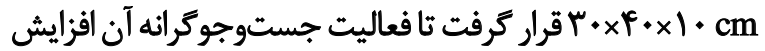

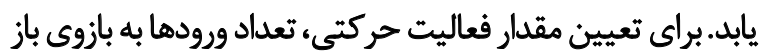

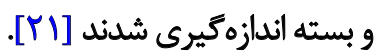

دادهها با استفاده از نسخه 19 نرمافزار آمارى SPS نسخه

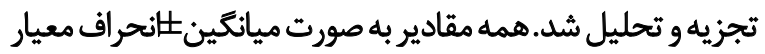

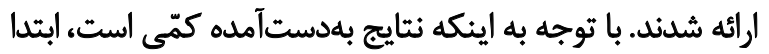

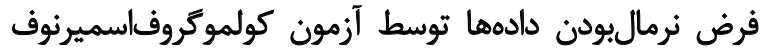

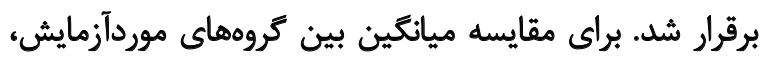
تحليل واريانس يكىطرفه و آزمون تعقيبى توكى و برانى مئين مقايسه

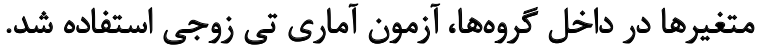

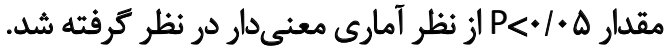

Ladigt

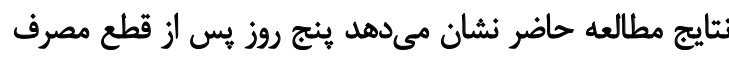

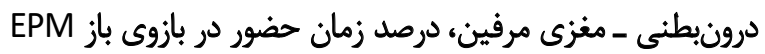

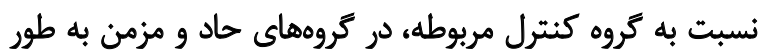

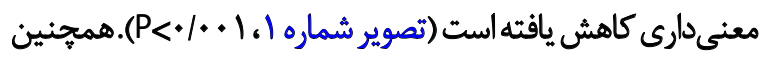

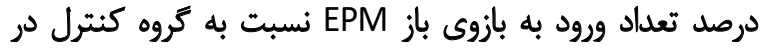

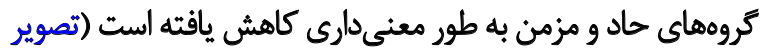

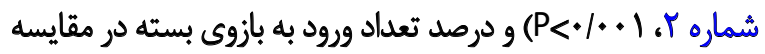
با كروه كنترل اختلاف معنى دارى نداشته است (تصوير شماره ب).

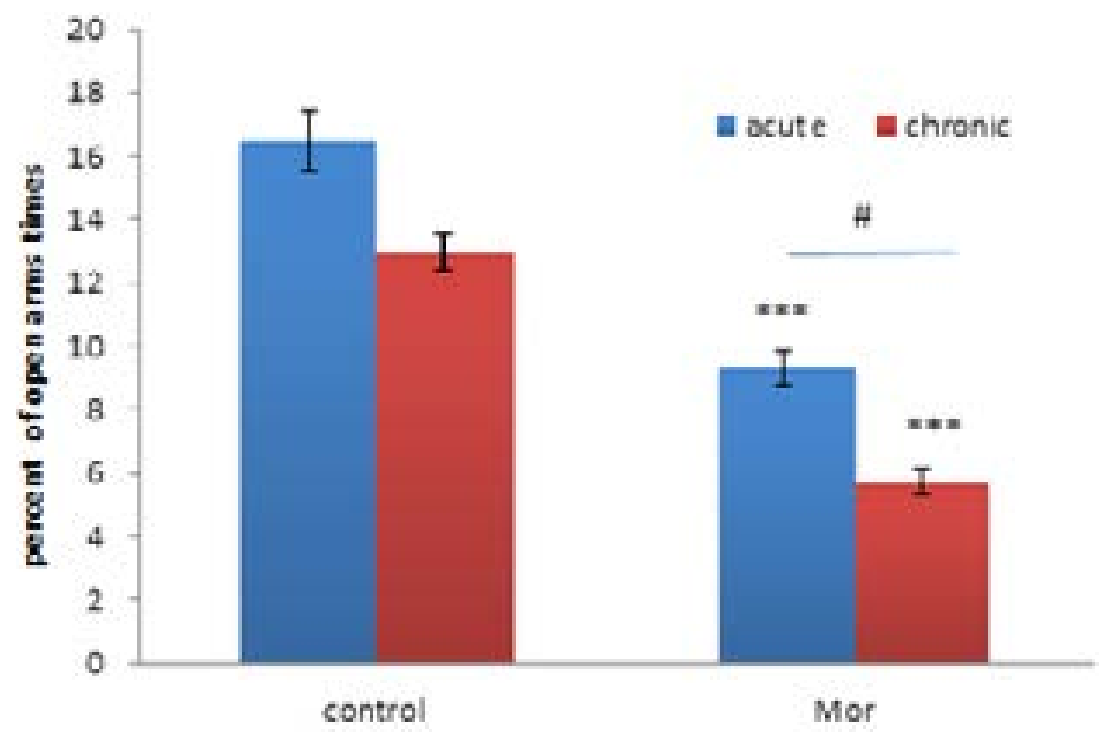

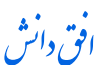

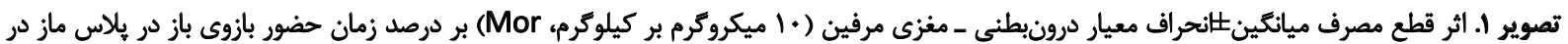

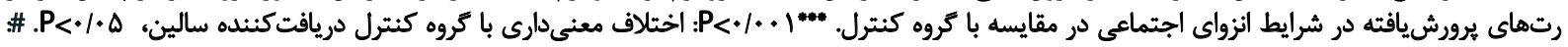

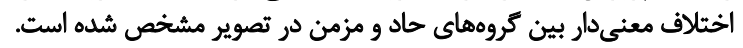




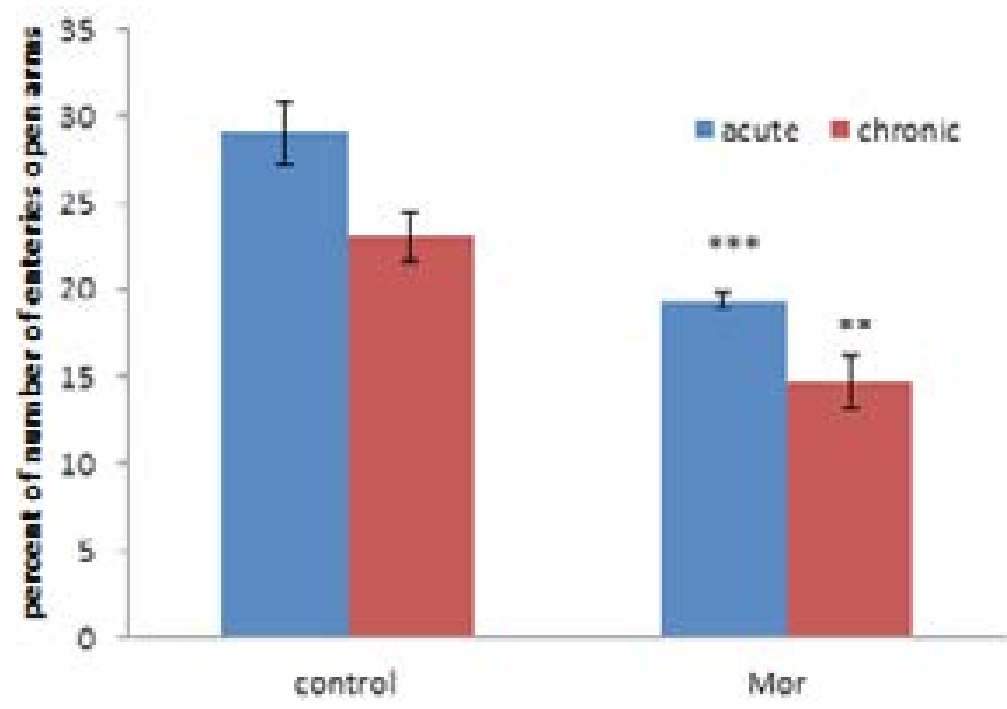

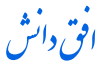

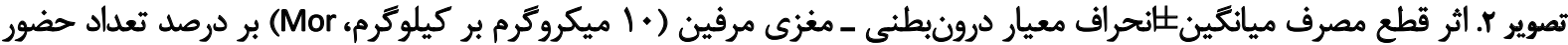

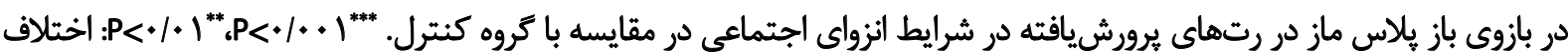

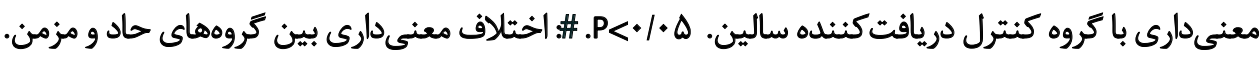

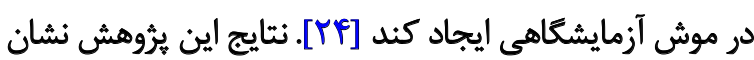

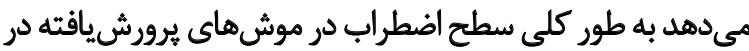
شرايط انزواى اجتماعى بالاتر بوده است.

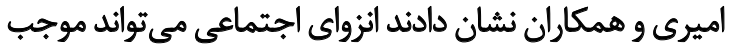

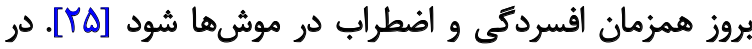

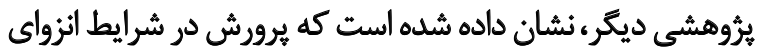

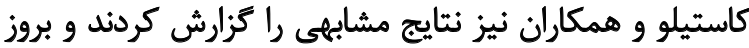

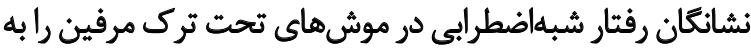

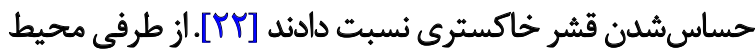

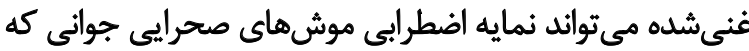

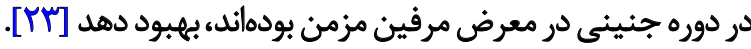

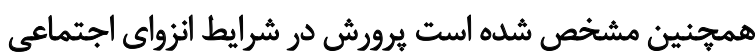

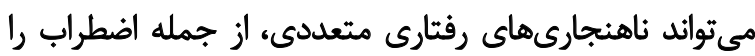

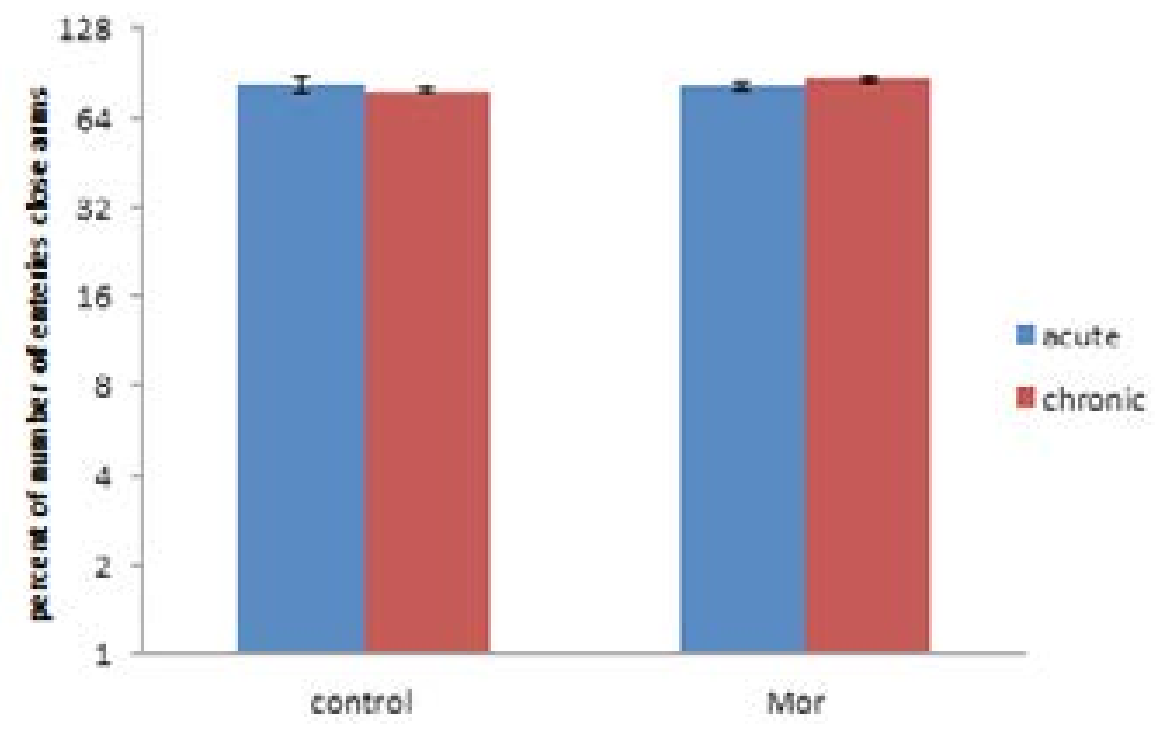

إنتواتث

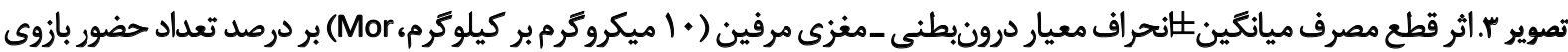

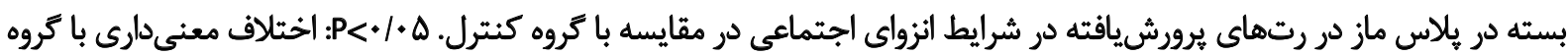

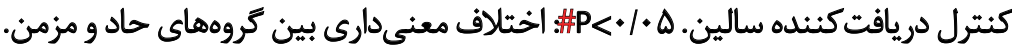


عاطفى منفى همراه با ترك حاد و مزمن مورفين شامل رفتار

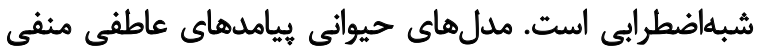

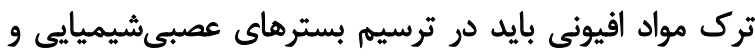

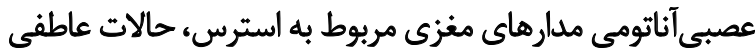
و و باداش مغز مفيد واقع شوند.

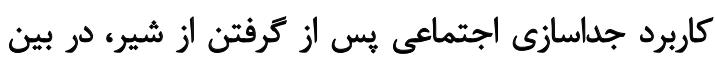

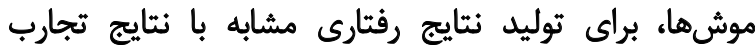

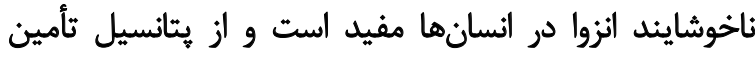

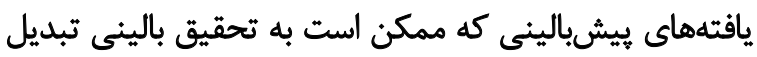

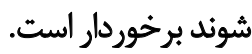

با توجه به اينكه تحقيقات كمى در اين زمينه روى موشهاي

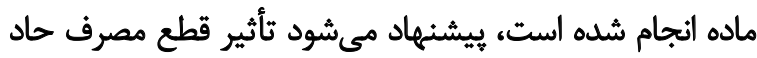

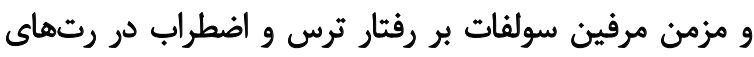

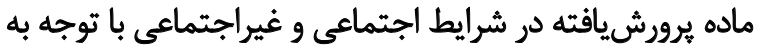
روشهاى مختلف تزريق، بررسى شود.

ملاحظات اخلاقي

\section{يبيووى أز أصول الخالاق بثوهش}

تمامى مراحل آزمايش بر اساس يروتكل تأييدشده كميتهاخلاق

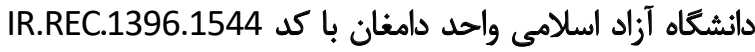

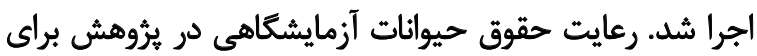

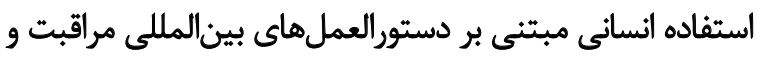
استفادهاز حيوائات آزمايشكاهي بوده است.

$$
\text { ماني مالى }
$$

اين مقاله حامى مالى ندارد.

مشاركت ثويسند مكان

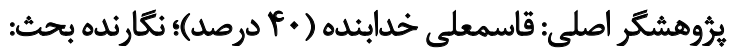

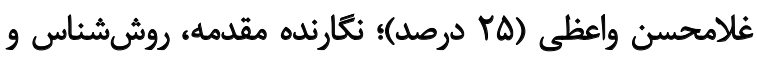

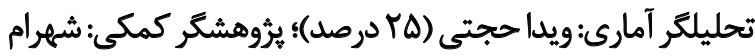
شرفى (·ا درصد).

$$
\text { تعارض مثأع }
$$

بنابر اظهار نويسندكان اين مقاله تعارض منافع ندارد.

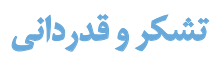

بدينوسيله نويسندكان مقاله از دانشكاه آزاد اسلامى واحد دامغان سياسكزارى و قدردانى مى كنيند.
اجتماعى و تنش ناشى از آن إسخكويى كيرنده إيبوئيد كايا

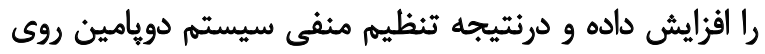

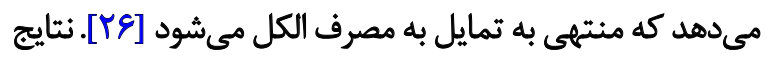

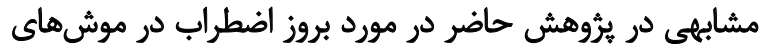

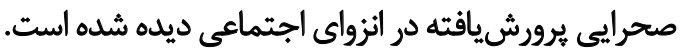

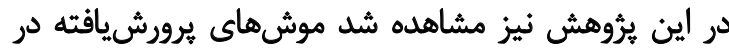

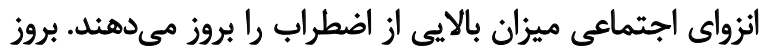

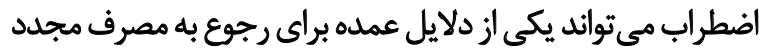

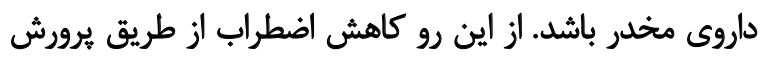

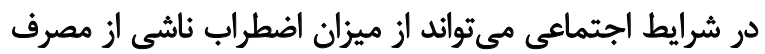

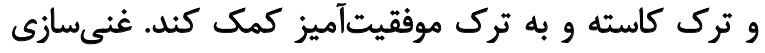

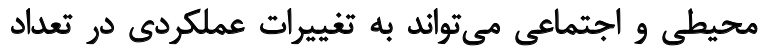

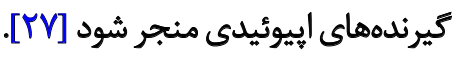
از آنجا كه انزواى اجتماعى باعث كاهش عملكرد مغز ،كاهش بردي

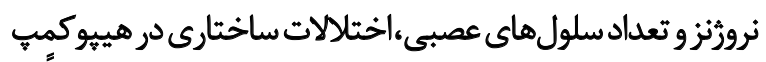

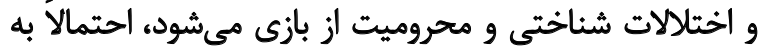

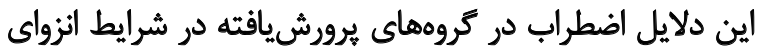

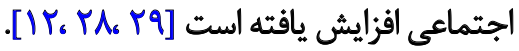

جايگاه اثرات مهم اييوئيدها درآميكدال است؛ به طورى كه

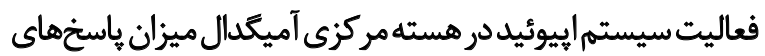

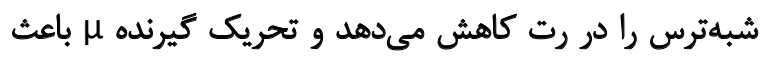

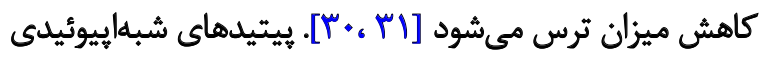

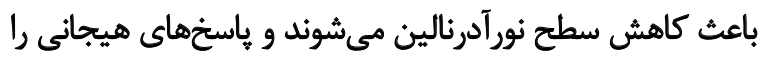

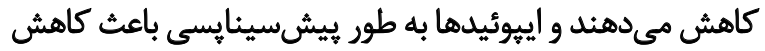

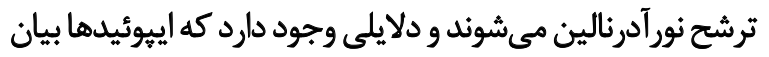

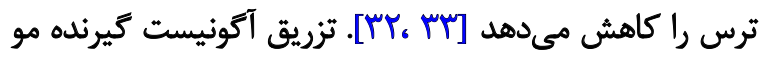

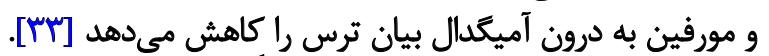

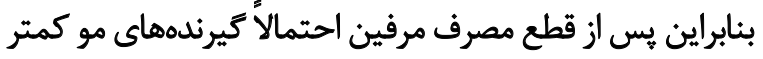

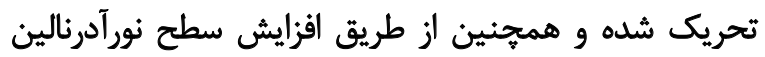

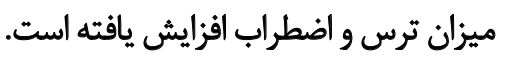
از طرف ديكر يكى از بيامدهاى مصرف مرفين، كاهش

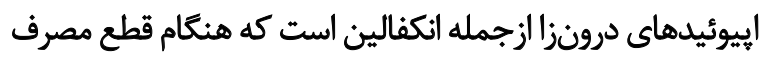

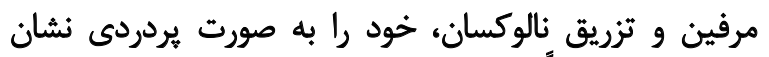

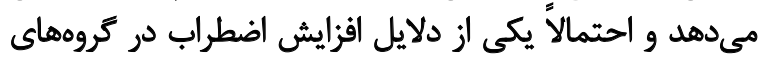

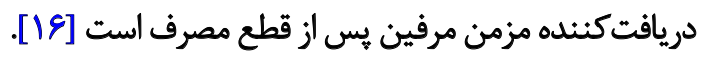

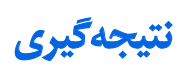

نتايج اين تحقيق نشان داد قطع تجويز درونبطنى ـمغزى حاد

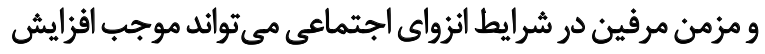

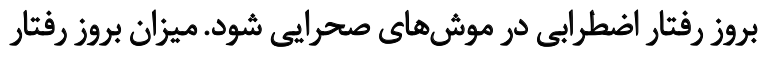

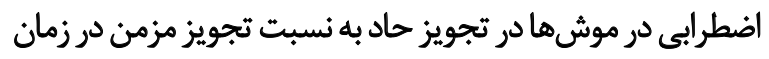

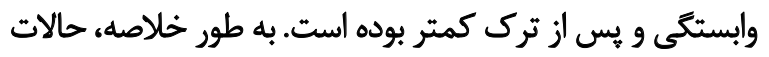




\section{References}

[1] Webster R, editor. Neurotransmitters, drugs and brain function. Hoboken: John Wiley \& Sons; 2001. [DOI:10.1002/0470846577]

[2] Volkow ND, Koob GF, McLellan AT. Neurobiologic advances from the brain disease model of addiction. New England Journal of Medicine. 2016; 374(4):363-71. [DOI:10.1056/NEJMra1511480] [PMID] [PMCID]

[3] Katzung BG. Basic and clinical pharmacology. New York: McGraw-hill; 2009.

[4] Kest B, Palmese CA, Hopkins E, Adler M, Juni A. Assessment of acute and chronic morphine dependence in male and female mice. Pharmacology Biochemistry and Behavior. 2001; 70(1):149-56. [DOI:10.1016/S00913057(01)00600-1]

[5] Mamiya T, Noda Y, Ren X, Hamdy M, Furukawa S, Kameyama T, et al. Involvement of cyclic AMP systems in morphine physical dependence in mice: Prevention of development of morphine dependence by rolipram, a phosphodiesterase 4 inhibitor. British Journal of Pharmacology. 2001; 132(5):1111-7. [DOI:10.1038/sj.bjp.0703912] [PMID] [PMCID]

[6] Bartoletti M, Gaiardi M, Gubellini C, Bacchi A, Babbini M. Morphine attenuation of a conditioned emotional response in post-dependent rats. European Journal of Pharmacology. 1990; 185(2-3):163-7. [DOI:10.1016/0014-2999(90)90636-K]

[7] Miladi-Gorji H, Rashidy-Pour A, Fathollahi Y. Anxiety profile in morphine-dependent and withdrawn rats: Effect of voluntary exercise. Physiology \& Behavior. 2012; 105(2):195-202. [DOI:10.1016/j.physbeh.2011.08.010] [PMID]

[8] Grasing K, Wang A, Schlussman S. Behavioral measures of anxiety during opiate withdrawal. Behavioural Brain Research. 1996; 80(1-2):195201. [DOI:10.1016/0166-4328(96)00035-6]

[9] Harris AC, Gewirtz JC. Elevated startle during withdrawal from acute morphine: A model of opiate withdrawal and anxiety. Psychopharmacology. 2004; 171(2):140-7. [DOI:10.1007/s00213-003-1573-0] [PMID]

[10] Vachon P, Millecamps M, Low L, Thompsosn SJ, Pailleux F, Beaudry F, et al. Alleviation of chronic neuropathic pain by environmental enrichment in mice well after the establishment of chronic pain. Behavioral and Brain Functions. 2013; 9(1):22. [DOI:10.1186/1744-9081-9-22] [PMID] [PMCID]

[11] Han X, Wang W, Xue X, Shao F, Li N. Brief social isolation in early adolescence affects reversal learning and forebrain BDNF expression in adult rats. Brain Research Bulletin. 2011; 86(3-4):173-8. [DOI:10.1016/j.brainresbull.2011.07.008] [PMID]

[12] Beard RL, O'Connor MK. Listening to Alzheimer's: The role of social location in illness narratives. Pennsylvania: IGI Global; 2015.

[13] Famitafreshi HR, Karimian M. Reduction of neurogenesis with social isolation decreases pain sensitivity in tail flick test in male rats. World Journal of Neuroscience. 2017; 7(2):223-31. [DOI:10.4236/ wjns.2017.72018

[14] Van den Berg CL, Kitchen I, Gerrits MA, Spruijt BM, Van Ree JM. Morphine treatment during juvenile isolation increases social activity and opioid peptides release in the adult rat. Brain Research. 1999; 830(1):1623. [DOI:10.1016/S0006-8993(99)01330-X]

[15] Van den Berg CL, Van Ree JM, Spruijt BM, Kitchen I. Effects of juvenile isolation and morphine treatment on social interactions and opioid receptors in adult rats: Behavioural and autoradiographic studies. European Journal of Neuroscience. 1999; 11(9):3023-32. [DOI:10.1046/j.14609568.1999.00717.x] [PMID]
[16] Gupta RK, Bruehl S, Burns JW, Buvanendran A, Chont M, Schuster E et al. Relationship between endogenous opioid function and opioid analgesic adverse effects. Regional Anesthesia \& Pain Medicine. 2014 39(3):219-24. [DOI:10.1097/AAP.0000000000000083] [PMID] [PMCID]

[17] Vanderschuren $\amalg$, Stein EA, Wiegant VM, Van Ree JM. Social isolation and social interaction alter regional brain opioid receptor binding in rats. European Neuropsychopharmacology. 1995; 5(2):119-27. [DOI:10.1016/0924-977X(95)00010-M]

[18] Bianchi M, Fone KF, Azmi N, Heidbreder CA, Hagan JJ, Marsden CA. Isolation rearing induces recognition memory deficits accompanied by cytoskeletal alterations in rat hippocampus. European Journal of Neuroscience. 2006; 24(10):2894-902. [DOI:10.1111/j.1460-9568.2006.05170.x] [PMID]

[19] Tzschentke TM, Kögel BY, Frosch S, Linz K. Limited potential of cebranopadol to produce opioid-type physical dependence in rodents. Addic tion Biology. 2018; 23(5):1010-9. [DOI:10.1111/adb.12550] [PMID]

[20] Pellow S, Chopin P, File SE, Briley M. Validation of open: Closed arm entries in an elevated plus-maze as a measure of anxiety in the rat. Journal of Neuroscience Methods. 1985; 14(3):149-67. [DOI:10.1016/01650270(85)90031-7]

[21] Ookawa K, Mochizuki K, Shida E, Suzuki T, Ooba T, Matumoto T, et al. Anti-anxiety effect of ovary lipid extracted from skipjack tuna (Katsuwonus pelamis) in rats. Journal of Veterinary Medical Science. 2007; 69(6):633-6. [DOI:10.1292/jvms.69.633] [PMID]

[22] Castilho VM, Borelli KG, Brandao ML, Nobre MJ. Anxiety-like symptoms induced by morphine withdrawal may be due to the sensitization of the dorsal periaqueductal grey. Physiology \& Behavior. 2008; 94(4):552-62. [DOI:10.1016/j.physbeh.2008.03.014] [PMID]

[23] Ahmadalipour A, Sadeghzadeh J, Vafaei AA, Bandegi AR, Mohammad khani R, Rashidy-Pour A. Effects of environmental enrichment on behavioral deficits and alterations in hippocampal BDNF induced by prenatal exposure to morphine in juvenile rats. Neuroscience. 2015; 305:372-83. [DOI:10.1016/j.neuroscience.2015.08.015] [PMID]

[24] Koike H, Ibi D, Mizoguchi H, Nagai T, Nitta A, Takuma K, et al. Behavioral abnormality and pharmacologic response in social isolation-reared mice. Behavioural Brain Research. 2009; 202(1):114-21. [DOI:10.1016/j. bbr.2009.03.028] [PMID]

[25] Amiri S, Haj-Mirzaian A, Rahimi-Balaei M, Razmi A, Kordjazy N, Shirzadian $A$, et al. Co-occurrence of anxiety and depressive-like behaviors following adolescent social isolation in male mice; possible role of nitrergic system. Physiology \& Behavior. 2015; 145:38-44. [DOI:10.1016/j. physbeh.2015.03.032] [PMID]

[26] Karkhanis AN, Rose JH, Weiner JL, Jones SR. Early-life social isolation stress increases kappa opioid receptor responsiveness and downregulates the dopamine system. Neuropsychopharmacology. 2016 41(9):2263-74. [DOI:10.1038/npp.2016.21] [PMID] [PMCID]

[27] Smith MA, Chisholm KA, Bryant PA, Greene JL, McClean JM, Stoops $W W$, et al. Social and environmental influences on opioid sensitivity in rats: Importance of an opioid's relative efficacy at the mu-receptor. Psychopharmacology. 2005; 181(1):27-37. [DOI:10.1007/s00213-005 2218-2] [PMID]

[28] Porkess MV. The impact of social isolation on rat behaviour [PhD dissertation]. Nottingham: University of Nottingham; 2008.

[29] Coudereau JP, Stain F, Drion N, Sandouk P, Monier C, Debray M, et al. Effect of social isolation on the metabolism of morphine and its passage through the blood-brain barrier and on consumption of sucrose solutions. Psychopharmacology. 1999; 144(3):198-204. [DOI:10.1007/ s002130050994] [PMID] 
[30] McNally GP, Pigg M, Weidemann G. Opioid receptors in the midbrain periaqueductal gray regulate extinction of pavlovian fear conditioning. Journal of Neuroscience. 2004; 24(31):6912-9. [DOI:10.1523/JNEUROSCI.1828-04.2004] [PMID] [PMCID]

[31] Barke KE, Hough LB. Morphine-induced increases of extracellular histamine levels in the periaqueductal grey in vivo: A microdialysis study. Brain Research. 1992; 572(1-2):146-53. [DOI:10.1016/00068993(92)90463-J]

[32] Sanders MJ, Kieffer BL, Fanselow MS. Deletion of the mu opioid receptor results in impaired acquisition of Pavlovian context fear. Neurobiology of Learning and Memory. 2005; 84(1):33-41. [DOI:10.1016/j. nlm.2005.03.001] [PMID]

[33] Heidbreder CA, Weiss IC, Domeney AM, Pryce C, Homberg J, Hedou G, et al. Behavioral, neurochemical and endocrinological characterization of the early social isolation syndrome. Neuroscience. 2000; 100(4):74968. [DOI:10.1016/50306-4522(00)00336-5] 